\section{A shifting balance}

Disruption of the normal pattern of imprinting has been implicated in cancer; for example, loss of imprinting (LOI) at the insulin-like growth factor 2 (IGF2) locus is seen in several common cancers, and the $10 \%$ of the population with LOI at IGF2 have a higher than normal risk of developing colorectal cancer. A recent report by Andrew Feinberg and colleagues shows that epigenetic alterations to IGF2 promote intestinal tumorigenesis and that a shift towards an undifferentiated cell fate might contribute to an increased rate of tumour initiation.

Wanting to understand how epigenetic changes at the IGF2 locus cause intestinal tumorigenesis, the authors created a mouse model using a deletion of the differentially methylated region (DMR) that regulates Igf2 imprinting in mice. Female mice lacking the DMR inherit a normal active copy of Igf2 from their father, but also an abnormally active (imprint-free) copy of these females with adenomatous polyposis coli $(A p c)$-mutant males predisposes the resulting offspring to multiple intestinal neoplasia. The authors compared Apc mutants carrying a silenced maternal copy of Igf2 (they called these LOI(-) mice) to their Apc mutant littermates (LOI(+) mice) that had two active copies of $\operatorname{Ig} f 2$.

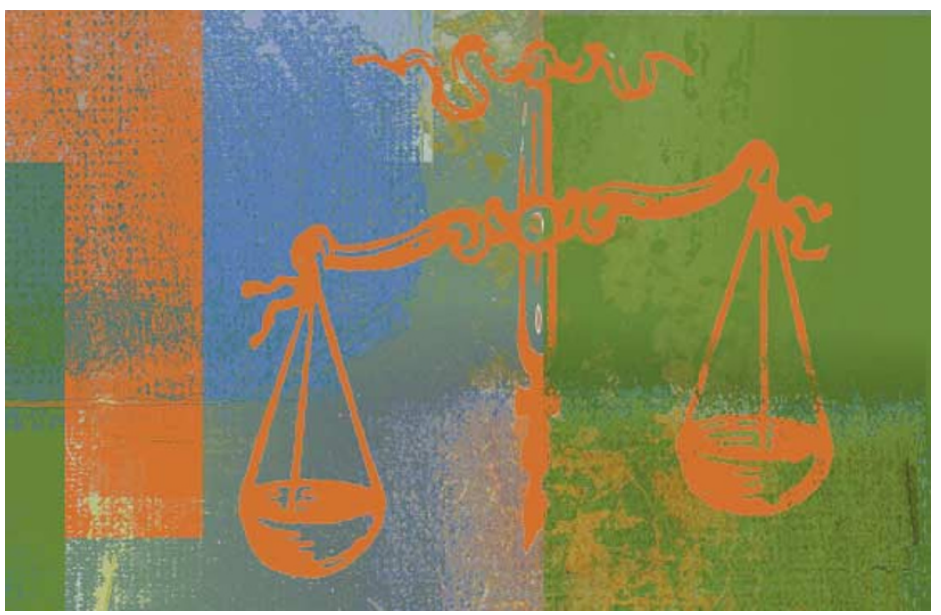

Confirming the role of Igf 2 in colon cancer, $\mathrm{LOI}(+)$ mice developed twice as many tumours in the small intestine and colon. Curiously however, LOI(+) mice also had longer intestinal crypts - the invaginations in the intestinal epithelium, at the bottom of which stem-cell proliferation occurs. Feinberg and co-workers postulated that this increase in crypt length could be due to a shift in the ratio of differentiated to undifferentiated epithelial cells. Sure enough, $\mathrm{LOI}(+)$ mice had higher levels of undifferentiated epithelial-cell markers in their small intestines than LOI(-) mice. A similar increase was seen in the intestinal epithelium of patients with IFG2 LOI.

The authors suggest that this shift increases tumour initiation, rather than the rate of tumour progression, and, supporting this, they found that $\mathrm{LOI}(+)$ and LOI(-) mice have the same ratio of small to large tumours. It is likely that the shift in normal tissue to a less differentiated state increases the number of cells that are vulnerable to subsequent genetic alterations. Future work will determine whether epithelial differentiation or LOI itself will be the better predictor of cancer risk.

Jenny Bangham

(2) References and links ORIGINAL RESEARCH PAPER Sakatani et al. Loss of imprinting of lfg2 alters intestinal 24 Feb 2005 (doi: 10.1126/science.1108080) maturation and tumorigenesis in mice. Science Igf2 from their mother. Crossing

\section{TRIAL WATCH}

\section{At risk}

Inhibitors of cyclooxygenase-2 (COX2) have been heralded as new, effective therapies for both pain relief and cancer prevention. However, recent findings indicate that COX2 inhibitors, when used long term, significantly increase the risk of death from heart attack or stroke.

The identification of COX2 as a promoter of intestinal tumorigenesis prompted trials of $\mathrm{COX} 2$ inhibitors as potential chemopreventive agents. But the voluntary withdrawal of the COX2 inhibitor rofecoxib (Vioxx) last September encouraged a closer inspection of COX2-inhibitor-related cardiovascular events.

The Adenomatous Polyp Prevention on Vioxx (APPROVe) trial was designed to evaluate whether 3 years of treatment with rofecoxib would reduce the risk of recurrent adenomatous polyps among patients with a history of colorectal adenomas. Because inhibition of COX2 suppresses the anti-thrombotic molecule prostacyclin, but not the pro-thrombotic molecule thromboxane $\mathrm{A}$, an assessment of cardiovascular events by an independent committee was integral to the APPROVe trial. Their findings prompted the termination of this study 2 months ahead of schedule, when a total of 1,287 patients had received $25 \mathrm{mg}$ of rofecoxib daily and 1,299 patients had been given a placebo for a mean of 2.4 years and 2.6 years respectively. Serious thrombotic events - heart attacks or strokes - were reported in 121 patients. Compared with the placebo group, the rofecoxib group had an increased risk of confirmed thrombotic events (relative risk 1.92; 95\% confidence interval, 1.19 to 3.11 ). Interestingly, in a post hoc analysis, this difference between the 2 groups was only evident in the second 18 months of the trial and might help to explain why other, shorter rofecoxib trials had not reported any increase in cardiovascular risk.

The Adenoma Prevention with Celecoxib (APC) trial was ongoing when the APPROVe study was terminated, causing an independent investigation into the cardiovascular safety of celecoxib in the APC trial. Previous clinical trails and observational studies with celecoxib had not clearly identified an increased risk of cardiovascular events.

Patients in the APC trial were treated with placebo or $200 \mathrm{mg}$ or $400 \mathrm{mg}$ of celecoxib twice daily. A total of 2,035 patients were assessed, $77 \%$ of whom had completed the study. Compared with the placebo group, the group taking $200 \mathrm{mg}$ twice daily had a hazard ratio for death from cardiovascular events of 2.3 (95\% confidence interval, 0.9 to 5.5 ) and those taking $400 \mathrm{mg}$ twice daily had a hazard ratio of 3.4 (95\% confidence interval, 1.4 to 7.8). Interestingly, a preliminary safety committee evaluation of the Prevention of Spontaneous Adenomatous Polyps (preSAP) trial, where patients are receiving one $400 \mathrm{mg}$ dose of celecoxib once a day, showed no evident cardiovascular risk factors.

Both studies conclude that the use of COX2 inhibitors in preventing colorectal neoplasia needs to be carefully considered against the background of increased risk of death from cardiovascular-related events.

ORIGINAL RESEARCH PAPERS Solomon, S. et al. Cardiovascular risk associated with celecoxib in a clinical trial for colorectal adenoma prevention. N. Eng. J. Med. 15 Feb 2005 (doi:10.1056/NEJMoa050405) | Bresalier, R. S. et al. Cardiovascular events associated with rofecoxib in a colorectal adenoma chemoprevention trial. N. Eng. J. Med. 15 Feb 2005 (doi:10.1056/NEJMoa050493) 